\title{
Missed Sentinel Case of Naturally Occurring Pneumonic Tularemia Outbreak: Lessons for Detection of Bioterrorism
}

Zygmunt F. Dembek, PhD, Ronald L. Buckman, MD, Stephanie K. Fowler, MD, and James L. Hadler, MD

Background: Family physicians are likely to care for patients that have been exposed to diseases associated with bioterrorism. Persons with seemingly nondescript initial disease symptoms could be harbingers of a larger outbreak, whether naturally occurring or purposefully created.

Methods: We report a missed sentinel case of pneumonic tularemia associated with a naturally occurring outbreak. The patient's initial clinical symptoms and signs were nondescript, and the diagnosis was recognized by subsequent blood tests. The medical literature was searched using the key words "tularemia," "bioterrorism," "index of suspicion," and "sentinel case."

Results and Conclusions: Being alert to possible unexpected causes of a pneumonic summer illness in a patient with associated weight loss might have led to an earlier diagnosis of this sentinel case tularemia and its association with the subsequent outbreak. Individual patients are likely to visit a physician's office after a purposeful bioterrorism event. Greater efforts must be made to increase awareness in all primary care physicians who might see patients exposed to a bioterrorism illness. ( $\mathrm{J}$ Am Board Fam Pract 2003;16:339-42.)

Tularemia is a seldom-diagnosed zoonosis caused by the gram-negative coccobacillus Francisella tularensis. It is a highly infectious bacterium with a low infectious dose and has been developed for use as a biological weapon. ${ }^{1}$ The ability of clinicians to suspect promptly and diagnose accurately the pneumonic and typhoidal forms has taken on new importance. In 2000 the second major outbreak of pneumonic tularemia in 32 years occurred on Martha's Vineyard, Mass. ${ }^{2,3}$ A Connecticut resident was one of two sentinel cases, which although successfully managed, was not initially suspected or diagnosed as tularemia. This report examines the clinical symptoms and signs of this case and their relevance in an era of enhanced physician alertness for diseases of bioterrorism.

\section{Methods}

We report a missed sentinel case of pneumonic tularemia associated with a naturally occurring out-

Submitted, revised, 2 April 2003

From the Connecticut Department of Public Health (ZFD, JLH), Hartford, and Bolton Family \& Sports Medicine (RLB, SKF), Bolton, Conn. Address reprint requests to Zygmunt F. Dembek, PhD, Connecticut Department of Public Health, 410 Capitol Ave, MS \#11EPI, PO Box 340308, Hartford, CT 06134-0308. break. The patient's initial clinical symptoms and signs were nondescript, and the diagnosis was made by subsequent blood tests. The medical literature was searched using the key words "tularemia," "bioterrorism," "index of suspicion," and "sentinel case."

\section{Case Report}

On 5 June 2000, a 57-year-old man visited his family physician with complaints of fever, fatigue, anorexia, rhinorrhea, and some chest congestion of 7 days' duration. On 29 May 2000, he had the onset of illness with irritated eyes, rhinorrhea, anorexia, fever, and a feeling of fatigue. In addition, he initially had polydipsia and uncontrollable diarrhea that lasted for 2 days. After his diarrheal illness subsided, he developed intermittent right-sided head and neck pain, along with persistent anorexia. He reported a severe weight loss of 20 pounds (approximately 10\% body weight) during the first 5 days of illness. He did not seek medical care during this time; rather, he self-medicated with attapulgite (Kaopectate) and acetaminophen. He had no preexisting medical condition and was not a smoker.

At examination, his temperature was $102.8^{\circ} \mathrm{F}$, blood pressure $124 / 76 \mathrm{mmHg}$, and pulse $108 / \mathrm{min}$. He appeared mildly ill but had no rash. There were 
benign findings of the eyes, ear, nose and throat, no lymphadenopathy, clear lungs, normal respiration rate, normal cardiac signs, benign abdominal findings, and normal neuromuscular findings. Clinical laboratory findings disclosed a white cell count of $7,800 / \mu \mathrm{L}$, with a differential count of $83 \%$ segmented neutrophils and $8 \%$ lymphocytes. He had an erythrocyte sedimentation rate of $55 \mathrm{~mm} / \mathrm{h}$, normal values for electrolytes, blood urea nitrogen, creatinine, and thyroid-stimulating hormone, and a negative Lyme antibody screening test. His chest radiograph showed right middle-lobe infiltrate consistent with a right middle-lobe bronchopneumonia. He was given clarithromycin $500 \mathrm{mg}$ twice daily for 10 days and ibuprofen and acetaminophen for pain.

The patient was a resident of Connecticut but had a second home in Martha's Vineyard, Mass, where he visited during the 3 days before the onset of symptoms (26 May to 28 May 2000). Activities performed during his stay included performing yard work and mowing his lawn with a side discharge mower. He was also aware of multiple dog ticks (Dermacentor variabilis) crawling on his body during his stay but did not notice any deer ticks (Ixodes scapularis) or tick bites.

Although his symptoms resolved without further medical treatment, subsequent media publicity about the outbreak of tularemia on Martha's Vineyard prompted him to telephone the Connecticut Department of Public Health (CDPH) on 1 August 2000, to inquire about the possibility that he had contracted tularemia during his stay there. His primary care physician was contacted, and a blood test for tularemia was requested. Testing performed at Quest Diagnostic Laboratory, Wallingford, Conn, showed a tularemia antibody titer 1:1280. Because the patient had been completely asymptomatic for some time, no further treatment was deemed necessary. The diagnostic results confirming probable tularemia prompted the immediate electronic notification of all Connecticut hospital-based infectious disease physicians to be alert for tularemia, especially among patients with links to Martha's Vineyard.

\section{Discussion}

Tularemia is endemic throughout much of the northern hemisphere. Approximately 200 cases of tularemia are reported annually in the United
States, mostly from rural areas in south-central and western states. ${ }^{1,4}$ Various small mammals are natural reservoirs of infection for $F$ tularensis, which they acquire through insect vectors or from environmental sources. Most human cases of tularemia are consequently associated with the bites of infective ticks and biting flies or with handling infected small mammals (primarily rabbits). ${ }^{1,4}$ Tularemia cases mostly occur in June through September and are likely greatly underreported. Endemic inhalational tularemia cases in the United States are considered to be attributable to the more virulent $F$ tularensis biovar tularensis (type A) and often follow an acute and severe course, with prominent pneumonitis. ${ }^{1,4}$

This report describes one of the two sentinel cases in an outbreak of tularemia on Martha's Vineyard. Tularemia is listed as a category A pathogen by the Centers for Disease Control and Prevention with respect to its potential for use as an agent of bioterrorism. ${ }^{5}$ The sentinel cases of pneumonic tularemia were harbingers of a larger naturally occurring event, but under other circumstance, they could have been the first cases seen from an exposure produced as an act of bioterrorism. Fortunately, this outbreak was associated with lawn mowing or brush cutting, an activity in which this person engaged during his stay on Martha's Vineyard. ${ }^{3}$

The primary clinical signs and symptoms of tularemia vary in severity according to virulence of the infecting organism, dose, and site of inoculum. Acute onset usually occurs after a presumably dosedependent incubation period of 1 to 21 days (average 3-5 days). Various schemes have been proposed to classify clinical manifestations of tularemia. ${ }^{1,4,6}$ Evans and colleagues ${ }^{4}$ suggest that tularemia can be thought of as two syndromes: ulceroglandular and typhoidal. In ulceroglandular tularemia (approximately $75 \%$ of cases) the pathogen appears to be well contained by a vigorous inflammatory reaction, pneumonia is less common, and the patient's prognosis is good. It is characterized by fever, chills, headache, malaise, an ulcerated skin lesion, and painful regional lymphadenopathy. ${ }^{4,6}$

This Connecticut case most likely had typhoidal-pneumonic tularemia, which could result from an aerosol exposure to $F$ tularensis. Typhoidal tularemia (approximately $25 \%$ of naturally acquired cases) often manifests in the patient with fever and weight loss, but not lymphadenopathy. ${ }^{4,6}$ Typhoi- 
dal tularemia is particularly difficult to diagnose clinically and usually is seen as an atypical pneumonia without cutaneous or mucosal membrane lesions or regional lymphadenitis. ${ }^{4,6}$ Pneumonic tularemia is a severe atypical pneumonia that can be fulminant with a high case fatality rate if untreated. It can be the primary manifestation after inhalation of organisms. Pneumonic tularemia is seen in 30\% to $80 \%$ of the typhoidal cases. ${ }^{4,6}$

After potential exposure or diagnosis, early treatment is recommended with an antibiotic from the tetracycline (such as doxycycline) or fluoroquinolone (such as ciprofloxacin) class, which are taken orally, or the antibiotics streptomycin or gentamicin, which are given intramuscularly or intravenously. ${ }^{1,6}$ Sensitivity testing of the tularemia bacterium can be done in the early stages of a response to determine which antibiotics would be most effective. Isolation or quarantine of tularemia patients is not required, since human-to-human transmission does not occur. ${ }^{1,6}$ Standard precautions are appropriate for the care of patients with draining lesions or pneumonia. A vaccine for tularemia used in the past to protect laboratory workers is currently under review by the Food and Drug Administration. ${ }^{1,6}$

In this sentinel case, a patient with tularemia sought care from his family physician in Connecticut rather than a hospital clinic at Martha's Vineyard. He had a prolonged but moderating illness that was successfully treated on an outpatient basis. Further diagnostic evaluation was not immediately done. These factors made disease diagnosis and detection difficult, because it was not quickly associated with the subsequent cluster of patients in Martha's Vineyard. Given the nondescript symptoms of this patient, the lack of enhanced suspicion for bioterrorism diseases, and the lack of an alert about or local cluster of patients with similar illness, there was no compelling reason to suspect tularemia.

The patient reported his illness to the CDPH after illness resolution as a result of media publicity from the Massachusetts outbreak. At the time of his illness (early June 2000), Connecticut physicians had no heightened awareness of patient exposure to tularemia and would not have been expected to make this diagnosis. A single patient coming to a physician's office in the absence of additional epidemiologic information could occur with a purposeful bioterrorism event in which tularemia is used. The 2000 Martha's Vineyard pneumonic tularemia outbreak was initially detected by hospital clinical staff on Martha's Vineyard who were looking for tularemia-caused pneumonic summer illness (Dennis L. Hoak, MD, personal communication, August 28, 2002).

Although this Connecticut case of tularemia was not diagnosed initially, a cluster of patients with similar symptoms should be recognized as an abnormal event that should initiate notification of local and state health authorities and further diagnostic testing. During this naturally occurring outbreak, discrete disease exposures likely occurred, with a lapse of 10 days between the sentinel cases and a subsequent cluster of ill persons and additional cases evolving for 5.5 months.

A purposeful biological attack using aerosolized tularemia would likely result in point source exposures. Depending upon the size of the release, there might be a larger number of simultaneous cases. A likely result would be an abrupt onset of large numbers of acutely ill persons, with the rapid progression from upper respiratory tract symptoms and bronchitis to life-threatening pleuropneumonitis and systemic infection. Such a situation would be noticed. The discovery of these symptoms in healthy children and adults should also alert health care providers and public health authorities to the possibility of bioterrorism. As with this sentinel case of a naturally occurring tularemia outbreak, ill persons might seek treatment from their primary health care provider rather than go to a hospital emergency department.

An attempted bioterrorist attack that resulted in only one or several cases of pneumonic tularemia might well go without notice, however, as this case would have without a heightened sense of awareness. To best detect initial cases of a disease associated with bioterrorism, there must be an enhanced awareness along with the prudence to order the appropriate laboratory diagnostic test. If Connecticut health care providers had been notified previously of enhanced surveillance by $\mathrm{CDPH}$ for summer pneumonic illness with associated weight loss, this Connecticut case might have prompted a call to CDPH in early June 2000. The subsequent epidemiologic investigation might have determined that there was an out-of-state potential exposure to tularemia much more rapidly and prompted earlier statewide, regional, and national notifications for 
alert to this disease. In Connecticut, health alerts were not sent out until late August 2000.

Because tularemia is a potential agent of bioterrorism, health care workers have been required to report to CDPH the clinical and laboratory finding of tularemia since 1 January 1999. To heighten awareness for bioterrorism diseases at Connecticut hospitals, $\mathrm{CDPH}$ has conducted clinician training for the past 3 years. As this case showed, however, patients might seek care at locations other than hospitals, including physician offices and walk-in clinics. Greater efforts need to be made to increase awareness, not only among hospital emergency department clinicians and infectious disease specialists, but among all primary care physicians, because they all might see patients exposed to a bioterrorism illness.

Standard laboratory diagnosis is based upon serologic antibody detection with an agglutination test. It can be positive 2 to 3 weeks after the infection. ${ }^{8}$ A fourfold change in agglutinin antibody titer is diagnostic, although a single titer of $1: 160$ or greater indicates recent infection, and both private and government laboratory results can be obtained in 48 hours. $^{9}$ Presumptive laboratory identification of the causative organism using routine procedures is time consuming and could take several weeks, and it should not be relied upon for patient treatment. The CDPH laboratory currently has the ability to culture $F$ tularensis from a clinical specimen or bacteriological isolate, perform the slide agglutination assay, and will shortly have the capability to perform both polymer chain reaction (PCR) testing and rapid antigen detection of $F$ tularensis. ${ }^{10}$ Both PCR and time-resolved fluorescence technologies hold the promise of having test results available within an 8-hour workday.

State and local health departments need to publicize diagnostic procedures for all agents of bioterrorism, especially for class A bioterrorism pathogens. Physician training regarding illnesses associated with bioterrorism can be obtained from courses offered by Centers for Disease Control and
Prevention, ${ }^{11}$ the US Army Medical Research Institute of Infectious Diseases, ${ }^{12}$ and other agencies.

We thank Kevin Griffith, MD, Epidemic Intelligence Service, Centers for Disease Control and Prevention, for helpful review of the manuscript.

\section{References}

1. Dennis DT, Inglesby TV, Henderson DA, et al. Tularemia as a biological weapon: medical and public health management. JAMA 2001;285:2763-73.

2. Teutsch SM, Martone WJ, Brink EW, et al. Pneumonic tularemia on Martha's Vineyard. N Engl J Med 1979;301:826-8.

3. Feldman KA, Enscore RE, Lathrop SL, et al. An outbreak of primary pneumonic tularemia on Martha's Vineyard. N Engl J Med 2001;345:1601-6.

4. Evans ME, Gregory DW, Schaffner W, McGee ZA. Tularemia: a 30-year experience with 88 cases. Medicine (Baltimore) 1985;64:251-69.

5. Public Health Emergency Preparedness \& Response. Biological diseases/agents. Available at: http://www. bt.cdc.gov/Agent/agentlist.asp.

6. Kortepeter M, Christopher G, Cieslak T, et al, editors. Medical management of biological casualties handbook, 4th ed. Washington, DC: Government Printing Office, 2001.

7. Fatality intensifies inquiry into cases of rare disease. Hartford Courant. September 2, 2000.

8. Koskela P, Salminen A. Humoral immunity against Francisella tularensis after natural infection. J Clin Microbiol 1985;22:973-9.

9. Bryant KA. Tularemia: Lymphadenitis with a twist. Pediatric Ann 2002;31:189-90

10. Johnasson A, Berglund L, Eriksson U, et al. Comparative analysis of PCR versus culture for diagnosis of ulceroglandular tularemia. J Clin Microbiol 2000; 38:22-6.

11. PHPPO strives to attain its vision through a focus on the public health system. Public Health Practice Program office, Centers for Disease Control and Prevention. Available at: http://www.phppo.cdc.gov/ PHTN/default.asp; http://www.bt.cdc.gov/VideoArchive.asp.

12. United States Army Medical Research Institute of Infectious Diseases (USAMRIID). Available at: http:// www.usamriid.army.mil/education/index.html. 